\title{
A NOTE FOR SOLVING THE EXPRESSION EQUATION BY THE METHOD OF MOMENT*
}

\author{
TAKAO SATOH AND KUNIO ATSUMI \\ Dept. of Chem. Eng., Faculty of Eng., Univ. of \\ Shizuoka, Hamamatsu
}

\footnotetext{
In the previous report ${ }^{3)}$ the method of moment was employed to solve the basic differential equation of forced expression. No explanation, however, was given as to the validity of using the larger root $\boldsymbol{\beta}$ for the non-dimensional critical time $\boldsymbol{\theta}_{c r i}$.

It is shown in this complementary report that theoretically the value of $\beta$ is restricted to the larger root of Eq. $(17)^{3)}$ with the range of $0<K<\sqrt{3 / 2}$

Some comments on the diffusion process with different boundary conditions are also given.
}

In the previous report ${ }^{37}$ the value of $\beta$, which is the non-dimensional critical time $\theta_{c r i}$, was determined as the larger positive root of the quadratic equation:

$$
24 F(0) \alpha \beta^{2}+(\alpha-108 F(0)) \beta+3 / 2=0
$$

without any discussion about the choice.

Some discussion about the choice of $\beta$ by the original investigator $\mathrm{H}$. Fujita was reproduced in the text by Crank ${ }^{22)}$, and recently Ames ${ }^{11}$ stated that it was not so rigorous.

It is the purpose of this report to discuss the problem because the Ames description is not clear.

From Eq. $\left(17^{\prime}\right)^{3)}$, one obtains

$$
\sqrt{\beta}=\left(1 \pm \sqrt{1-2 K^{2} / 3}\right) / 4 K
$$

where $K$ is defined by Eq. $\left(26^{\prime \prime}\right)^{3)}$ and is the non-dimensional form of $K_{e}$.

In Fig. 1 the square of the larger $\sqrt{\beta}$ of Eq. (A) is shown by the line $(A)_{L}$ and the square of the smaller by the line $(A)_{s}$. These lines coincide at the point $\mathrm{P}$.

The physical meaning of $A_{2}$ is visualized from Eq. $(18)^{37}$ as

$$
\varepsilon(1, \theta)=1-A_{2}(\theta)
$$

That is, $A_{2}$ relates to $\varepsilon$ at $v=1$ in the later stage and it increases as time elapses. Consequently $\phi$ becomes positive and $\psi$ is clearly positive by definition (Eq. $\left.\left(19^{\prime}\right)^{32}\right)$.

On the other hand from Eqs. $(16)^{3)}$ and $\left(19^{\prime}\right)$

$$
q(1)=\alpha / 30
$$

Inserting Eq. (19") into Eq. $(20)^{3)}$ yields

$$
\phi(1)=14(\alpha \beta+\alpha / 12-9 / 2) / 15
$$

As $\phi(1)$ is positive, one obtains

$$
\alpha \beta+\alpha / 12-9 / 2>0
$$

Using Eq. $\left(26^{\prime}\right)^{3)}$ and noting that $K$ and $\beta$ must be positive from their physical meaning, the above relation becomes

$$
\beta^{3 / 2}-\frac{\beta}{2 K}+\frac{\sqrt{\beta}}{12}-\frac{1}{96 K}>0
$$

Inserting the larger $\sqrt{\beta}$ of Eq. (A) into Eq. $\left(20^{\prime \prime \prime}\right)$, one obtains

$$
\sqrt{1-2 K^{2} / 3}>0
$$

\footnotetext{
* Received on January 26, 1970
}

and therefore

$$
K<\sqrt{3 / 2}
$$

If the smaller $\sqrt{\beta}$ of Eq. (A) were inserted, the relation becomes $\sqrt{1-2 K^{2} / 3}<0$, and this is not allowed.

Thus it is clear that the value of $\beta$ must be the larger positive root of Eq. (17) and the range of $K$ is $0<K<$ $\sqrt{3 / 2}$.

The above restriction on $K$ comes solely from the boundary conditions $(9)^{3)}$ and $(10)^{3)}$ and does not depend on the method of moment.

The Irange of $\beta$ restricted by the relation $\left(20^{\prime \prime \prime}\right)$ is shown by the shaded area in Fig. 1 in which the broken line itself is to be excluded.

When the expression coefficient is constant, $\alpha$ is 30 from Eq. (16) and the roots of Eq. (17) are 1/12 and $1 / 40$, then the values of $K$ become 1.155 and 1.186 respectively from Eq. $\left(17^{\prime}\right)$. The set $(\beta=1 / 12, K=$ 1.155) satisfies the relation $\left(20^{\prime \prime \prime}\right)$ as shown by the point $Q$ in Fig. 1, but the set $(\beta=1 / 40, K=1.186)$ does not (cf. the point $R$ ). Therefore, the value of $\beta$ must be the larger positive root of Eq. (17).

The same procedure is applied for the diffusion process

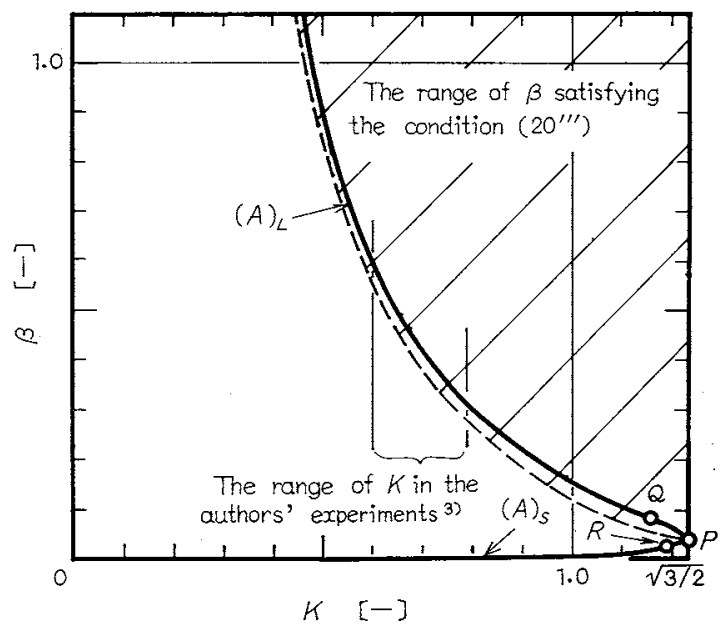

Fig. 1 The range of $\beta$ with varying $K$ 
with the following boundary conditions ${ }^{2 \mathrm{~b}\rangle}$ :

$$
\begin{array}{llr}
c=0, & t=0, & 0<x<1 \\
c=1, & x=1, & t>0 \\
\partial c / \partial x=0, & x=0, & t>0
\end{array}
$$

In this case $\sqrt{\beta}$ is found to be the larger root of Eq. $(9.228)^{2 \mathrm{a})}$, given as

$$
\sqrt{\beta}=\frac{1+\sqrt{1-2 K^{2} / 3 F(1)}}{4 K}
$$

and the required range of $K$ is $0<K<\sqrt{3 F(1) / 2}$.

\section{Notation}

$c=$ non-dimensional concentration of diffusing substance

$F(1)=$ non-dimensional coefficient of diffusion, $F(c)$, at $c=1$

$t=$ non-dimensional time of diffusion process

$x=$ non-dimensional distance in opposite direction of diffusion from phase boundary

Note: The other symbols are the same as those in the previous report ${ }^{3)}$

\section{Literature cited}

1) Ames, W. F.: "Nonlinear Partial Differential Equations in Engineering”, 254 (1965), Academic Press, New York and London

2a) Crank, J.: "The Mathematics of Diffusion", 180 (1956) Oxford Univ. Press, London

2b) Crank, J.: ibid., 178

3) Satoh, T. and K. Atsumi: J. Chem. Eng. Japan, 3, 92 (1970) 